\title{
Isolamento de lectinas por cromatografia de afinidade
}

\section{Aislamiento de lectinas por cromatografía de afinidad}

\author{
Isolation of lectins by affinity chromatography
}

\author{
José Camilo Torres Romero', Myriam Janeth Ortega Torres², \\ Jhon Alexander Infante Betancour ${ }^{3} \&$ Cicero Antonio Maia Cavalcante ${ }^{4}$

\begin{abstract}
${ }^{1}$ Licenciado en Biología, Magister en Ciencias - Bioquímica, Doctor en Bioquímica. ${ }^{2}$ Licenciada en Biología. Magister en Producción Animal- Genética Molecular Animal. Estudiante Doctorado en Producción Animal. Universidad Nacional de Colombia. Bogotá. Colombia. ${ }^{3}$ Biólogo, Magister en Ciencias-Biología. ${ }^{4 B}$ Bacharel em Ciências Farmacêuticas, Licenciatura em Ciências Biológicas, Especialização em Ensino de Biologia, Especialização em Saúde Pública, Mestrado profissional em Ensino de Ciências e Matemática.
\end{abstract} \\ ${ }^{1,2}$ Escuela de Ciencias Agrícolas, Pecuarias y del Medio Ambiente -ECAPMA. Universidad Nacional Abierta \\ y a Distancia UNAD. Bogotá. Colombia. ${ }^{3} Y$ OLUKA ONG Fundación de Investigación en Biodiversidad \\ y Conservación. ${ }^{4}$ Instituto Federal do Ceará - IFCE. Jaguaribe -Ceará, Brasil.
}

1jose.torres@unad.edu.co, ${ }^{2}$ myriam.ortega@unad.edu.co, 3ihhon.infante@yoluka.org.co, ${ }^{4}$ cicero.carla@uol.com.br

\section{Resumo}

Cromatografia de afinidade é uma técnica usada para separar compostos, como, por exemplo, determinadas proteínas, que têm a capacidade de se ligar não covalentemente e reversivelmente a moléculas específicas conhecidas como ligantes. Esse método difere das técnicas de cromatografia clássica devido a proteína conseguir ser separada com base em uma única propriedade bioquímica. Em cromatografia de afinidade, o ligante está ligado covalentemente à matriz, que deve ser quimicamente inerte, porosa e também ter uma variedade de grupos funcionais adequados para acoplamento com ligantes diferentes. Várias matrizes e ligantes são usados em cromatografia de afinidade, dependendo da proteína a ser purificada. Esse trabalho descreverá alguns aspectos importantes para o isolamento de lectinas através da técnica de cromatografia de afinidade utilizando-se carboidratos.
Palavras-chave: proteínas, lectinas, carboidratos, isolectinas, cromatografia.

\section{Resumen}

La cromatografía de afinidad es una técnica utilizada para separar compuestos de muestras complejas, tales como ciertas proteínas, que tienen la capacidad de unirse de forma reversible y no covalente con moléculas específicas conocidas como ligando. Este método difiere de las técnicas de cromatografía clásicas de proteína para realizar el aislamiento en la base de una única propiedad bioquímica. En la cromatografía de afinidad el ligando se une covalentemente a la matriz, que debe ser químicamente inerte, porosa y, además, tener una variedad de grupos funcionales adecuados para el acoplamiento con diferentes enlazadores. Varias matrices y condiciones se utilizan en cromatografía de afinidad, dependiendo de la proteína a purificar. Este artículo 
de investigación describe algunos aspectos importantes para aislar lectinas por cromatografía de afinidad utilizando carbohidratos.

Palabras clave: proteínas, lectinas, carbohidratos, isolectinas, cromatografía.

\section{Abstract}

Affinity chromatography is a technique used to separate compounds from complex samples, such as certain proteins, that have the ability to bind reversibly and non-covalently with specific molecules known as ligands. This method differs from classical protein chromatography techniques by performing isolation at the base of a single biochemical property. In affinity chromatography the ligand is covalently bound to the matrix, which must be chemically inert, porous and, furthermore, have a variety of functional groups suitable for coupling with different linkers. Several matrices and conditions are used in affinity chromatography, depending on the protein to be purified. This paper describes some important aspects for isolating lectins by affinity chromatography using carbohydrates.

Key-words: proteins, lectins, carbohydrates, isolectin, chromatography.

\section{Introdução}

Os métodos de separação de misturas de diferentes componentes mediante a utilização de fases são conhecidos como cromatografia (Cardona \& Muñoz, 2016). O início da utilização da cromatografia como método de separação data de 1903, ocorrendo o seu posterior desenvolvimento e evolução a partir de 1930. A primeira pessoa a definir o termo cromatografia foi o botânico russo Miguel Tswett (18721913) no ano de 1906, escolhendo esse termo das palavras gregas khromatos (cor) e graphein (escrita) para descrever a separação dos pigmentos de plantas em diferentes zonas coloridas. Atualmente, o termo cromatografía tem sido utilizado para o isolamento também de compostos incolores, permitindo o isolamento de proteínas através de sua afinidade por outras biomoléculas. Esse trabalho tem como objetivo demonstrar a importância da cromatografia de afinidade para o isolamento de lectinas.

\section{Características das lectinas importantes para seu isolamento}

As lectinas são um grupo de proteínas que tem como característica principal a capacidade de ligarse a carboidratos (Micucci \& Camps, 1987). Elas estão presentes em todos os organismos, o que sugere que desempenham diversas funções biológicas básicas, tais como regulação, adesiva, defesa contra patógenos, dentre outras. (Varki et al., 2009). Um organismo apresenta, normalmente, um número de diferentes lectinas presentes em várias isoformas, chamadas isolectinas (Van Damme et al., 1998). As isolectinas se diferenciam na sua estrutura primária, apresentando pequenas variações nessa estrutura, e na especificidade de ligação ao carboidrato, podendo apresentar diferenças em suas atividades biológicas (Leavitt et al., 1977). Portanto, ao se isolar uma lectina também é importante pensar nas suas isolectinas.

A estrutura geral das lectinas permite a sua classificação em três grandes grupos. O primeiro representado pelas Merolectinas que apresentam um único domínio de ligação de hidrato de carbono. O segundo representado pelas Hololectinas que apresentam dois ou mais domínios de ligação de hidratos de carbono. O terceiro representado pela Quimerolectina que contêm domínios adicionais não-lectinicos, geralmente catalíticos (Peumans \& Van Damme, 1995). A maioria das lectinas apresentam múltiplos locais de ligação que podem ser uteis em associações de entrecruzamento com glicorreceptores específicos na superfície da célula (Geijtenbeek \& Gringhuis, 2016). Lectinas também atuam como moléculas de reconhecimento nas interações de molécula-celula (Brown, Crocker, 2016), célula-celula (Geijtenbeek \& Gringhuis, 2016) e estão envolvidas em processos celulares, incluindo adesão, 
migração, diferenciação, proliferação e apoptose (Huang et al., 2017). Além disso, muitas lectinas ativam respostas fisiológicas diversas em vários organismos e possuem propriedades imunomoduladoras, apresentando funções potenciais no câncer e na metástase (Perillo et al., 1998; Sharon \& Lis, 1989, Wu et al., 2017), o que as torna potencialmente úteis em aplicações biotecnológicas e biomédicas.

A ligação entre as lectinas e os carboidratos caracterizam-se pelo fato de serem não covalentes e reversíveis, sendo realizadas por interações hidrofóbicas, ligações de hidrogênio, interações eletrostáticas, interações de van der Waals e atração dipolo (Lehr, 2000). A ligação entre os açúcares -mono e dissacarídeos- e a lectina é relativamente fraca, com constates de dissociação expressas em micromolar ou milimolar. Por outro lado, as interações multivalentes da lectinas com complexos ramificados de hidratos de carbono resultam em ligações mais fortes com constantes de dissociação expressas em nanomolares ou mesmo picomolares (Varki et al., 2008).

Em geral, as lectinas possuem propriedades bioquímicas e de ligação, que são muito convenientes para sua purificação por cromatografia de afinidade utilizando carboidratos. Elas não reagem cataliticamente com eles, ao menos que a lectina tenha um subdomínio glicosidase catalítico. Como observado anteriormente, as lectinas se ligam aos hidratos de carbono de forma não covalente e reversível. Os ligantes mais utilizados para o isolamento das lectinas, são mono e dissacarídeos, que ligados de forma relativamente fraca, a essas proteínas são prontamente libertados de uma coluna de afinidade por eluição competitiva usando hidratos de carbono específicos libres ( Moreira et al., 1997; Moreira \& Perrone, 1975). Além disso, as lectinas e carboidratos são compostos geralmente estáveis, portanto eluição utilizando técnicas de condições extremas de $\mathrm{pH}$ e / ou força iónica podem também ser aplicados para libertar uma lectina a partir da coluna de afinidade (Vega \& Perez, 2006).
Qualquer fonte de interesse pode ser selecionada para o isolamento de lectinas, de seres humanos e outros animais, a plantas, fungos, bactérias e até mesmo os vírus (Procópio et al., 2017). Em primeiro lugar, uma lectina deve ser liberada a partir da fonte em solução, geralmente por liberação a partir das células. Vários métodos podem ser utilizados, dependendo sobre as características mecânicas do tecido de origem, como por homogeneização por ruptura mecânica ou por lise (Voet e Voet, 1995). As lectinas são proteínas solúveis em água, portanto, são extraídas com tampões aquosos e salinos.

A extração da lectina de interesse deve ser realizada a $4^{\circ} \mathrm{C}$ para evitar a sua desnaturação e degradação por enzimas proteolíticas. A adição de inibidores de protease no tampão de extração é recomendado se a atividade proteolítica é observada no extrato (Kvennefors et al, 2008; Matsumoto et al, 2011; Watanabe et al, 2007), apesar de algumas lectinas encontradas serem bastante resistentes à degradação proteolítica (Pohleven et al., 2009). Inibidores de glicosidase, tais como o inibidor de glucosidase -desoxinojirimicina $(0,2 \mathrm{mM})$ e do inibidor da manosidase -deoxymannojirimicin $(0,2 \mathrm{mM})$, também podem ser adicionados ao extrato para impedir a clivagem de hidratos de carbono imobilizados na coluna de afinidade (Watanabe et al., 2007). Antes do extrato ser aplicado a coluna de afinidade, algumas impurezas podem ser removidas usando precipitação fracionada com sulfato de amónio e / ou de outras técnicas cromatográficas clássicas. As frações de lectina podem ser monitoradas em todo o processo de purificação acompanhando-se a sua atividade hemoaglutinante, para isso utiliza-se de eritrócitos específicos, visto que, só as células vermelhas com glicanos lectinas específicas são aglutinados. (Cavalcante et al., 2016).

\section{Isolamento de Lectinas através da técnica de Cromatografía de Afinidade}

Uma vez preparado o extrato que exibe atividade de hemoaglutinação, o método de cromatografia de afinidade de hidratos de carbono adequado para a purificação da lectina deve ser selecionado. Isso envolve o adsorvente que pode se ligar a lectina e separá-lo a partir do extrato, e de uma técnica 
de eluição que possa liberar a lectina ligada a coluna. Por conseguinte, é importante conhecer a especificidade e estabilidade da lectina e também examinar a estabilidade do adsorvente, extremos de $\mathrm{pH}$ e temperatura podem ser utilizados para o acoplamento do ligante com o agente de eluição (Melgarejo et al., 2005). A especificidade da lectina desejada em um extrato pode ser determinada através da análise da sua atividade de aglutinação, utilizando várias classes de eritrócitos que expõem diversos glicanos em suas superfícies. Por exemplo, no sistema $A B O$, o grupo sanguíneo humano A expressa a forma não redutora do carboidrato $\mathrm{N}$-acetilgalactosamina, o grupo $\mathrm{B}$, expressa galactose e o grupo $O$ expressa fucosil-galactose (Schenkel-Brunner, 2007). Além disso, a inibição a aglutinação utilizando vários hidratos de carbono ou glicoproteínas, pode ser usado para determinar o especificidade da lectina, permitindo que o adsorvente lectina específica possa ser selecionado. A estabilidade da lectina pode ser avaliada examinando-se a atividade de aglutinação do extrato. No entanto, diversas observações (Moreira et al., 1997), sugerem que a lectina de um extrato é mais estável do que a isolada, provavelmente pela importancia dos acucares no contexto molecular da proteina (Abrantes et al., 2013).

Dependendo do ligante e do adsorvente, pode-se utilizar as técnicas de eluição competitiva ou eluição a diferentes $\mathrm{pH}$ e / ou força iónica para que a lectina ligada a coluna de afinidade seja liberada. Ao escolher a técnica mais apropriada para desadsorver uma lectina ligada, a especificidade, a sua estabilidade, e o tipo de adsorvente, isto é açúcar ou polissacarídeo complexo, devem ser considerados. Quando um monossacarídeo ou dissacarídeo é usado como um ligante, a lectina é facilmente liberada da coluna de afinidade por eluição competitiva, para isso devem ser utilizados hidratos de carbono específicos com maior afinidade para a lectina do que o adsorbente (Moreira \& Perrone, 1975).

A maioria dos autores relatam o uso de eluição competitiva de lectinas utilizando mono ou dissacárideo específico. Por exemplo, quando uma lectina foi isolada em Sepharose, galactose foi utilizado para a dessorção (Cao et al., 2010; DeSimone et al., 2006), enquanto que a glucose foi utilizada com Sephadex (Rittidach et al., 2007; Sun et al., 2007). As concentrações de açúcares utilizados são geralmente de 0,2 M (variando entre 0,01 - 0,5 $\mathrm{M})$, enquanto que o gradientes de açúcar também têm sido utilizados (Cammarata et al., 2007; Mansour \& Abdul-Salam, 2009). Posteriormente, estas lectinas associadas a açúcares devem ser tratadas para libertar seus sítios de ligação, geralmente por diálise (Vega \& Pérez, 2006). Alternativamente, a fim de evitar o último passo, as lectinas podem ser dessorvido da coluna com alteração das condições a extremos de $\mathrm{pH}$ ou de força iónica (Vega \& Pérez, 2006). Esta última técnica depende da estabilidade química das substâncias ligantes da matriz e adsorvida e não é adequado para lectinas e adsorventes que são destruídos em tais condições (Vega \& Pérez, 2006). Apesar dos hidratos de carbono e lectinas, geralmente, serem moléculas estáveis, é preciso ter cuidado para não danifica-los de forma irreversível (Obando, Barahona \& Chamorro, 2014). As frações contendo as proteínas devem ser neutralizados imediatamente, geralmente com $2 \mathrm{M}$ ou $1 \mathrm{M}$ de Tris- $\mathrm{HCl}$, pH 7,5 (Vega \& Pérez, 2006). A coluna também deve ser equilibrada com o tampão de ligação. Quando oligo ou polissacarídeos ramificados com elevada avidez para as lectinas são utilizados como adsorventes, as lectinas não pode ser prontamente eluída utilizando açúcares monovalentes com menor afinidade para lectinas. Neste caso, devem ser utilizados $\mathrm{pH}$ extremo e / ou condições de força iónica. Vários autores relataram a eluição de lectinas utilizando ácidos $(20-100 \mathrm{mM}$ de glicina- $\mathrm{HCl}$ ou alanina, $\mathrm{pH}$ 2,5 , ou $1-3 \%$ de ácido acético ácido) (Kaur et al., 2006) ou bases ( $\mathrm{NaOH} 10 \mathrm{mM}$, trietanolamina 0,1 $\mathrm{M}, \mathrm{pH}$ 11, ou Tris-OH tampão de pH 11,4 (Vega \& Pérez, 2006 ), assim como soluções de tampões contendo $\mathrm{NaCl} 1 \mathrm{M}$ e $3 \mathrm{M}$ de $\mathrm{MgCl} 2$ (gradiente foi também utilizado) (Naganuma et al., 2006). Em alguns casos, as lectinas foram eluídas utilizando 6 M (Matsumoto et al., 2011) ou 8M de Ureia (Suseelan et al., 2002). A atividade de ligação da lectina para carboidratos pode depender de íons metálicos bivalentes tal como acontece como as lectinas tipo C (Gringhuis et al., 2007; Den Dunnen et al., 
2008), que podem ser eluídas utilizando tampões contendo agentes quelantes, tais como 2-100 mM de ácido etilenodiaminotetracético (EDTA) (Ourth et al., 2005).

\section{Outros métodos para a purificação de lectinas}

Além de cromatografia de afinidade com os carboidratos, outros métodos têm sido utilizados para purificar lectinas. Algumas lectinas foram purificadas utilizando precipitação fraccionada com sulfato de amónio como sal precipitante como indica Oliveira et al. (2002) e Vence et al. (2012), seguido por métodos clássicos, tais como cromatografia de troca iónica e cromatografia de filtração em gel (Horibe et al, 2010; Pan et al, 2010). As lectinas são geralmente glicoproteínas, portanto, alguns autores descrevem seu isolamento por cromatografia de afinidade a partir da utilização das lectinas na captura de outra, por exemplo, a Concanavalina A é frequentemente imobilizada para isolar outras lectinas (Absar et al, 2005.; Charungchitrak et al, 2011; Konkumnerd et al, 2010; Petnual et al, 2010; Yan et al, 2010). Além disso, pode-se trabalhar com partículas ferromagnéticas com polissacarídeo imobilizado preparados para o isolamento de lectinas (Porter et al., 1998). As propriedades magnéticas das partículas favorecem a lavagem das impurezas utilizando um campo magnético, e os açúcares são utilizados para libertar as lectinas e recuperar as partículas (Angeli et al., 2009; Kavunja et al., 2015). Um novo método para a purificação cromatográfica de afinidade de lectinas é a utilização de membranas com nanofibras glicosiladas, no qual, uma membrana de afinidade com glicose imobilizada mostrou forte e reversível capacidade para isolar lectinas (Che et al., 2011).

\section{Conclusões}

Pode-se concluir que a cromatografia de afinidade com carboidratos é, o método mais amplamente utilizado para a purificação de lectinas, apresentando-se como um método simples que tira proveito de suas propriedades específicas. No entanto, não existe um protocolo de aplicação geral e a seleção da matriz de ligação mais apropriada, da técnica de eluição e da ativação da matriz podem ser difíceis, devendo ser selecionadas de acordo com cada tipo de lectina que se pretende isolar.

\section{Literatura citada}

1. Abrantes, V. E. F., Matias da Rocha, B. A., Batista da Nóbrega, R., Silva-Filho, J. C., Teixeira, C. S., Cavada, B. S., ... Delatorre, P. (2013). Molecular Modeling of Lectin-Like Protein from Acacia farnesiana Reveals a Possible Anti-Inflammatory Mechanism in Carrageenan-Induced Inflammation. BioMed Research International, 2013, 253483. http://doi. org/10.1155/2013/253483

2. Absar, N., Yeasmin, T., Raza, M. S., Sarkar, S. K. \& Arisaka, F. (2005). Single Step Purification, Characterization and N-Terminal Sequences of a Mannose Specific Lectin from Mulberry Seeds. The Protein Journal, 24(6), 369-377.

3. Angeli, R., da Paz, N. V. N., Maciel, J. C., Araújo, F. F. B., Paiva, P. M. G., Calazans, G. M. T., Valente, A. P., Almeida, F. C. L., Coelho, L. C. B. B., Carvalho Jr., L. B., Silva, M. d. P. C. \& Correia, M. T. d. S. (2009). Ferromagnetic Levan Composite: An Affinity Matrix to Purify Lectin. Journal of Biomedicine and Biotechnology, 2009 (179106), 1110-1143.

4. Brown, G. \& Crocker, P. (2016). Lectin Receptors Expressed on Myeloid Cells. Microbiol Spectr, 4(5): doi: 10.1128/microbiolspec.MCHD-0036-2016.

5. Cammarata, M., Benenati, G., Odom, E. W., Salerno, G., Vizzini, A., Vasta, G. R. \& Parrinello, N. (2007). Isolation and characterization of a fish F-type lectin from gilt head bream (Sparus aurata) serum. Biochimica et Biophysica Acta, 1770 (1), 150-155.

6. Cao, X., Huo, Z., Lu, M., Mao, D., Zhao, Q., Xu, C., Wang, C. \& Zeng, B. (2010). Purification of lectina from larvae of the fly, Musca domestica, and in vitro antitumor activity in MCF-7 Cells. Journal of Insect Science, 10 (164), 1-13.

7. Cardona, J. \& Muñoz, J. (2016). Phytochemical variability between Colombian accessions of Lippia alba (Mill.) N.E. Brown. Revista de Investigación Agraria y Ambiental, 7 (2) julio-diciembre, 39-49. Recuperado de: http://hemeroteca.unad.edu.co/index.php/riaa/article/view/1556/1903

8. Cavalcante, M., Torres-Romero, J., Lobo, M., Moreno, F., Bezerra, L., Lima, D., Matos, J., Moreira, R. \& Monteiro-Moreira, A. (2016). A panel of glycoproteins as candidate biomarkers for early diagnosis and treatment evaluation of B-cell acute lymphoblastic leukemia. Biomark Res, 27;4:1. doi: 10.1186/s40364-0160055-6. eCollection 2016.

9. Charungchitrak, S., Petsom, A., Sangvanich, P. \& Karnchanatat, A. (2011). Antifungal and antibacterial activities of lectin from the seeds of Archidendron jiringa Nielsen. Food Chemistry, 126 (3), 1025-1032. 
10. Che, A.-F., Huang, X.-J. \& Xu, Z.-K. (2011). Polyacrylonitrile-based nanofibrous membrane with glycosylated surface for lectin affinity adsorption. Journal of Membrane Science, 366 (1-2), 272-277.

11. Den dunnen, j. et al. (2008). Innate signaling by C-type lectin DC-sign dictates immune responses. Cancer immunol immunother, 26:605-610.

12. De-Simone, S. G., Netto, C. C. \& Silva Jr., F. P. (2006). Simple affinity chromatographic procedure to purify galactoside binding lectins. Journal of Chromatography $B, 838$ (2), 135-138.

13. Geijtenbeek, T. \& Gringhuis, S. (2016). C-type lectin receptors in the control of T helper cell differentiation. Nature Reviews Immunology, 16: 433-44.

14. Gringhuis, s. et al. (2007). C-type lectin DC-Sign modulates toll-like receptor signaling via raf-1 kinasedependent acetylation of transcription factor NF-kB. Immunity, 26(5): 605-616.

15. Horibe, M., Kobayashi, Y., Dohra, H., Morita, T., Murata, T., Usui, T., Nakamura-Tsuruta, S., Kamei, M., Hirabayashi, J., Matsuura, M., Yamada, M., Saikawa, Y., Hashimoto, K., Nakata, M. \& Kawagishi, H. (2010). Toxic isolectins from the mushroom Boletus venenatus. Phytochemistry, 71 (5-6), 648-657.

16. Huang, M., Wang, L., Zhang, H., Yang, C., Liu, R., Xu, J., Jia, Z. \& Song, L. (2017). The sequence variation and functional differentiation of CRDs in a scallop multiple CRDs containing lectin. Developmental \& Comparative Immunology, 67: 333-339.

17. Kaur, M., Singh, K., Rup, P. J., Kamboj, S. S., Saxena, A. K., Sharma, M., Bhagat, M., Sood, S. K. \& Singh, J. (2006). A Tuber Lectin from Arisaema jacquemontii Blume with Antiinsect and Anti proliferative Properties. Journal of Biochemistry and Molecular Biology, 39 (4), 432-440.

18. Kavunja, H., Voss, P., Wang, J. \& Huang, X.(2015). Identification of lectins from metastatic cancer cells through magnetic glyconanoparticles. Israel journal of chemistry, 55(3-4): 423-436.

19. Konkumnerd, W., Karnchanatat, A. \& Sangvanich, P. (2010). A thermostable lectin from the rhizomes of Kaempferia parviflora. Journal of the Science of Food and Agriculture, 90 (11), 1920-1925.

20. Kvennefors, E. C. E., Leggat, W., Hoegh-Guldberg, O., Degnan, B. M. \& Barnes, A. C. (2008). An ancient and variable mannose-binding lectin from the coral Acropora millepora binds both pathogens and symbionts. Developmental and Comparative Immunology, 32 (12), 1582-1592.

21. Leavitt, R., Felsted, R. \& Bachur, N. (1977). Biological and biochemical properties of Phaseolus vulgaris isolectins. J Biol Chem, 10(9):2961-2966

22. Lehr, C. (2000). Lectine-mediated drug delivery: The second generation of bioadhesives, Journal of ControIled Release, 65: 19.

23. Mansour, M. H. \& Abdul-Salam, F. (2009). Characterization of fucosebinding lectins in rock- and mud-dweIling snails inhabiting Kuwait Bay. Immunobiology, 214 (1), 77-85.
24. Matsumoto, R., Shibata, T. F., Kohtsuka, H., Sekifuji, M., Sugii, N., Nakajima, H., Kojima, N., Fujii, Y., Kawsar, S. M. A., Yasumitsu, H., Hamako, J., Matsui, T. \& Ozeki, Y. (2011). Glycomics of a novel type-2 N-acetyllactosaminespecific lectin purified from the feather star, Oxycomanthus japonicus (Pelmatozoa: Crinoidea). Comparative Biochemistry and Physiology, Part $B, 158$ (4), 266-273.

25. Melgarejo, L., Vega, N. \& Perez, G. (2005). Isolation and characterization of novel lectins from Canavalia ensiformis and dioclea grandiflora mart. ex benth. seeds. braz. j. plant physiol, 17(3):315-324.

26. Micucci, H. \& Camps, E. (1987). Lectinas: Obtención, Estructura Química, Propiedades y Aplicaciones Diagnósticas y Farmacológicas. Acta Farm, 6(1):35-54.

27. Moreira, R. \& Perrone, J. (1975). Purification and partial characterization of a lectin from the seeds of Phaseolus vulgaris. Plant Physiology, 59: 783-787.

28. Moreira, R., Monteiro, A., Horta, A., Oliveira, J. \& Cavada, B. (1997). Isolation and characterization of Dioclea altissima var. megacarpa seed lectin. Phytochemistry, 46: 139-144.

29. Naganuma, T., Ogawa, T., Hirabayashi, J., Kasai, K., Kamiya, H. \& Muramoto, K. (2006). Isolation, characterization and molecular evolution of a novel pearl shell lectina from a marine bivalve, Pteria penguin. Molecular Diversity, 10 (4): 607-618.

30. Obando, A., Barahona, R. \& Chamorro, D. (2014). Producción de dos cepas de Frankia sp. aisladas de Alnus acuminata H.B.K. por fermentación fed-batch. Revista de Investigación Agraria y Ambiental, 5 (1) enerojunio, 81-92. Recuperado de: http://hemeroteca.unad. edu.co/index.php/riaa/article/view/925/916

31. Oliveira, S., Nascimento, A., lima, M. , leite, Y. \& Benevides, N. (2002). Purification and characterisation of a lectin from the red marine alga Pterocladiella capillacea (s.g. gmel.) santel. \& hommers. Revista brasil. bot., 25 (4): 397-403.

32. Ourth, D. D., Narra, M. B. \& Chung, K. T. (2005). Isolation of mannosebinding C-type lectina from Heliothis virescens pupae. Biochemical and Biophysical Research Communications, 335 (4), 1085-1089.

33. Pan, S., Tang, J. \& Gu, X. (2010). Isolation and characterization of a novel fucose-binding lectin from the gill of bighead carp (Aristichthys nobilis). Veterinary Immunology and Immunopathology, 133 (2-4), 154-164.

34. Perillo, N. L., Marcus, M. E. \& Baum, L. G. (1998). Galectins: versatile modulators of cell adhesion, cell proliferation, and cell death. Journal of Molecular Medicine, 76 (6), 402-412.

35. Petnual, P., Sangvanich, P. \& Karnchanatat, A. (2010). A lectin from the rhizomes of turmeric (Curcuma longa L.) and its antifungal, antibacterial, and glucosidase inhibitory activities. Food Science and Biotechnology, 19 (4), 907-916.

36. Peumans, W. J. \& Van Damme, E. J. M. (1995). Lectins as plant defense proteins. Plant Physiology, 109 (2): 347-352. 
37. Pohleven, J., Obermajer, N., Sabotič, J., Anžlovar, S., Sepčić, K., Kos, J., Kralj, B., Štrukelj, B. \& Brzin, J. (2009). Purification, characterization and cloning of a ricin B-like lectina from mushroom Clitocybe nebularis with antiproliferative activity against human leukemic $\mathrm{T}$ cells. Biochimica et Biophysica Acta, 1790 (3), 173-181.

38. Porter, j. robinson, J., pickup, R. \& Edward, E. (1998). An evaluation of lectin-mediated magnetic bead cell sorting for the targeted separation of enteric bacteria. journal of applied microbiology, 84:722-732.

39. Procópio, T.., Moura, C., Albuquerque, P., Gomes, F. S., Santos, N., Coelho, L., ... \& Napoleão, T.(2017). Antibacterial lectins: action mechanisms, defensive roles and biotechnological potential. Antibacterials: Synthesis, Properties and Biological Activities, Nova Science Publishers Inc., New York, 69-89.

40. Rittidach, W., Paijit, N. \& Utarabhand, P. (2007). Purification and characterization of a lectina from the banana shrimp Fenneropenaeus merguiensis hemolymph. Biochimica et Biophysica Acta, 1770 (1): 106-114.

41. Schenkel-Brunner, H. (2007). Blood group antigens, In: Comprehensive Glycoscience, Kamerling, J. P., Lee, Y. C., Boons, G.-J., Suzuki, A., Taniguchi, N. \& Voragen, A. G. J., pp. (343-372), Elsevier, 978-044-452-746-2, Oxford.

42. Sharon, N. \& Lis, H. (1989). Lectins as cell recognition molecules. Science, 246 (4927), 227-234.

43. Sun, J., Wang, L., Wang, B., Guo, Z., Liu, M., Jiang, K. \& Luo, Z. (2007). Purification and characterisation of a natural lectin from the serum of the shrimp Litopenaeus vannamei. Fish and Shellfish Immunology, 23 (2), 292-299.

44. Suseelan, K. N., Mitra, R., Pandey, R., Sainis, K. B. \& Krishna, T. G. (2002). Purification and characterization of a lectin from wild sunflower (Helianthus tuberosus L.) tubers. Archives of Biochemistry and Biophysics, 407 (2), 241- 247.
45. Van Damme, E. J. M., Peumans, W. J., Pusztai, A. \& Bardocz, S. (February 25, 1998). Handbook of plant lectins: Properties and biomedical applications, John Wiley \& Sons, 978-047-196-445-2, New York.

46. Varki, A., Cummings, R. D., Esko, J. D., Freeze, H. H., Stanley, P., Bertozzi, C. R., Hart, G. W. \& Etzler, M. E. (Eds.). (October 15, 2008). Essentials of glycobiology, Cold Spring Harbor Laboratory Press, 978-087-969770-9, New York.

47. Vega, N. \& Pérez, G. (2006). Isolation and characterisation of a Salvia bogotensis seed lectina specific for the Tn antigen. Phytochemistry, 67 (4), 347- 355.

48. Vence, L., Rivera, M., Osorio, Y. \& Castillo, A. (2012). Caracterización microbiológica y fisicoquímica de aguas subterráneas de los municipios de La Paz y San Diego, Cesar, Colombia. Revista de Investigación Agraria y Ambiental, 3 (2) julio-diciembre, 27-35. Recuperado de: http://hemeroteca.unad.edu.co/index.php/ riaa/article/view/953/948

49. Voet, D. \& Voet, J. G. (January 15, 1995). Biochemistry (Second Edition), John Wiley \& Sons, 0- 471-58651-X, New York.

50. Watanabe, T., Matsuo, I., Maruyama, J.-i., Kitamoto, K. \& Ito, Y. (2007). Identification and Characterization of an Intracellular Lectin, Calnexin, from Aspergillus oryzae Using N-Glycan Conjugated Beads. Bioscience, Biotechnology, and Biochemistry, 71 (11), 2688-2696.

51. Wu, B., Mei, S., Cui, L., Zhao, Z., Chen, J., Wu, T. \& Li, G. (2017). Marine Lectins DIFBL and HddSBL Fused with Soluble Coxsackie-Adenovirus Receptor Facilitate Adenovirus Infection in Cancer Cells BUT Have Different Effects on Cell Survival. Mar. Drugs, 15(73):1 - 13.

52. Yan, Q., Zhu, L., Kumar, N., Jiang, Z. \& Huang, L. (2010). Characterisation of a novel monomeric lectin (AML) from Astragalus membranaceus with antiproliferative activity. Food Chemistry, 122 (3), 589-595.
Conflicto de Intereses

Los autores declaran no tener ningún conflicto de intereses

Recibido: agosto 04 de 2016 Aceptado: agosto 26 de 2016 
